\title{
Partial or Complete Unloading of Skeletal Muscle Leads to Specific Alterations of Anabolic Signal Transduction.
}

\author{
COLLEEN L. O'REILLY, J WILLIAM DEAVER, MICHAEL P. WIGGS, FLORENCE \\ LIMA, JOSHUA M. SWIFT, ELIZABETH S. GREENE, SUSAN A. BLOOMFIELD and \\ JAMES D. FLUCKEY
}

Muscle Biology Lab; Health and Kinesiology; Texas A\&M University; College Station, TX.

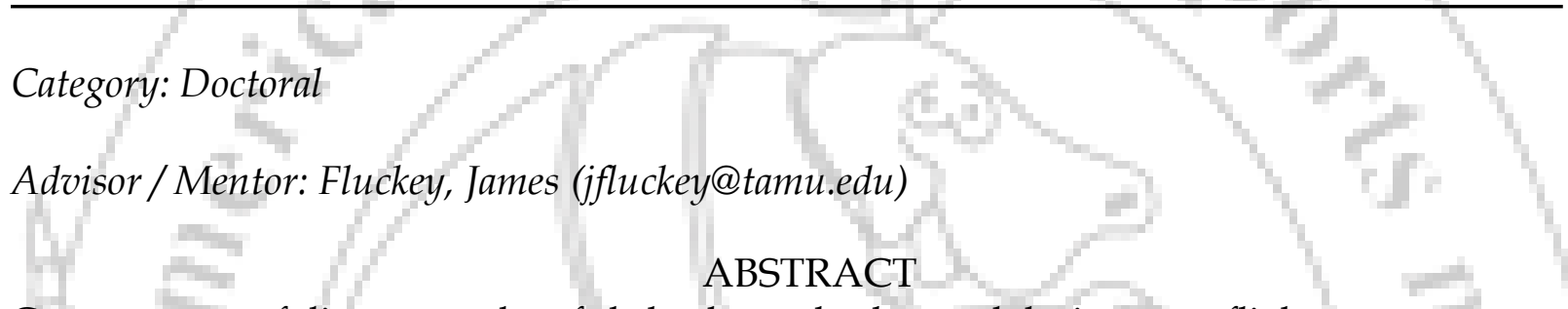

Consequences of disuse atrophy of skeletal muscle observed during spaceflight on astronaut health and performance are a focal point of space research. Decrements of both muscle mass and protein synthesis rates have been observed with exposure to varying muscle loading environments ( $1 \mathrm{G}>$ partial loading $>0 \mathrm{G}$ ), and most of the reduced muscle mass can be attributed to diminished rates of synthesis. However, specific mechanisms behind unloadingdependent reductions of protein synthesis are not well defined.

PURPOSE: To determine whether or not alterations of anabolic signal transduction was responsible for the changes previously observed in fractional synthesis rates with specific gravitational loading paradigms. METHODS: Female BALB/cByJ were normalized by bodyweight and assigned to normal cage ambulation $(1 \mathrm{G})$, partial weight bearing suspension titrated to approximately $33 \%$ bodyweight $(\mathrm{G} / 3)$, partial weight bearing titrated to $16 \%$ bodyweight $(\mathrm{G} / 6)$ and full unloading of hind limbs $(0 \mathrm{G})$ in specially designed cages. All mice were subjected to that loading environment for $21 \mathrm{~d}$ prior to tissue harvest, and monitored daily. Immunoblotting of the gastrocnemius $(n=23)$ was carried out to analyze alterations of anabolic signal transduction. Although numerous signaling intermediates were assessed, the focus of this abstract will be on ribosomal protein $\mathrm{S6}$ kinase (p70-S6K). This important protein has served as a marker of protein synthesis signal transduction as well as the anabolic capacity in skeletal muscle. RESULTS: Regardless of loading paradigm, no differences were detected among groups for the activation of p70-S6K (as indicated by the phospho: total protein content). Total protein content, however, was $\sim 27 \%$ lower than control in $0 \mathrm{G}$ and $\mathrm{G} / 3(\mathrm{P}=0.008)$ with $\mathrm{G} / 6$ not being different from control $(\mathrm{P}>0.05)$. CONCLUSION: In combination with previous data (unpublished observations), Partial gravitational fields at least partially rescues anabolic signaling, suggesting that a threshold level of stimulus is necessary to maintain anabolic capacity in muscle. These results may have important implications towards the development of strategies designed to counter the effects of partial/complete unloading on skeletal muscle based on how the anabolic capacity of muscle is affected. 